\title{
Sensorimotor Connectivity is related to Static Postural Control in Older Adults with History of Lateral Ankle Sprain
}

Katherine A. Bain ( $\nabla$ katherine.bain@uky.edu )

University of Kentucky

Kyle B. Kosik

University of Kentucky

Masafumi Terada

Ritusmeikan University

Phillip A. Gribble

University of Kentucky

Nathan F. Johnson

University of Kentucky

Research Article

Keywords: SMN, LAS, ROI, Ankle Sprain

Posted Date: April 28th, 2021

DOl: https://doi.org/10.21203/rs.3.rs-440912/v1

License: (c) (1) This work is licensed under a Creative Commons Attribution 4.0 International License.

Read Full License 


\section{Abstract \\ Context:}

The Sensorimotor Network (SMN) is often overlooked when determining relationships between postural control and sensorimotor function. SMN functional connectivity (FC) represents the temporal synchrony of functionally linked but spatially divergent brain regions. This study aimed to determine the relationship between SMN-FC and static postural control in older adults with a history of lateral ankle sprain (LAS).

\section{Methods}

Data were analyzed from twenty older adults (mean age $=67.0 \pm 4$.3yrs; 13 females) with a history of LAS. The SMN was identified from resting-state MRI data. Bilateral thalamic and post-central gyri regions of interest were identified. Balance was assessed for the involved and non-involved limbs via center of pressure velocity (COPV) in the medial-lateral (ML) and anterior-posterior (AP) directions.

\section{Results}

Contralateral SMN-FC was significantly associated with COPV_ML $(r=-0.468, P=.05)$ and COPV_AP $(r=$ $-0.530, P=.02)$ in the non-involved limb. No significant association was observed between involved limb balance and contralateral SMN-FC (COPV_ML: $r=-0.081, P=.75$; COPV_AP: $r=0.136, P=.60)$.

\section{Conclusion}

Findings suggest a dissociation between SMN-FC and balance in older adults with a history of LAS. The sensorimotor system's ability to communicate with itself after injury may have an impact on functional performance, such as balance.

\section{Introduction}

Lateral ankle sprains (LAS) are highly prevalent ${ }^{1,2}$, costly ${ }^{3}$, and often result in long-term negative health consequences. ${ }^{4}$ A large focus of LAS research has identified the pathomechanical, sensory-perceptual, motor-behavioral and subsequent functional impairments associated with LAS. ${ }^{5}$ However, it has been hypothesized that changes in the central nervous system (CNS) following a ligamentous injury may underlie the most commonly reported functional impairment, altered static postural control. ${ }^{6-9}$ Interestingly, altered static postural control is present in both injured and non-injured limbs, ${ }^{10}$ and can persist into late adulthood. ${ }^{11}$ Despite these findings, the underlying relationship between CNS communication and postural control in injured and non-injured limbs remains unknown. 
With the use of neuroimaging modalities, such as resting-state functional magnetic resonance imaging (fMRI), we are able to observe potential CNS-postural control relationships in individuals with a history of LAS. Resting-state fMRI is a noninvasive neuroimaging tool used to determine the temporal synchrony between functionally linked but spatially divergent brain regions, referred to as networks. ${ }^{12}$ The synchrony, or functional connectivity (FC), of the sensorimotor network (SMN) is an unexplored measure that may help to elucidate the relationship between central control and peripheral stability. Interestingly, activation of regions within this network are positively associated with greater balance performance ${ }^{13}$ and higher SMN connectivity may help to prevent future anterior cruciate ligament (ACL) ruptures. ${ }^{14}$ However, the relationship between SMN connectivity and postural control in injured and non-injured limbs remains unknown.

History of LAS is associated with deficits in both sensory and motor aspects of sensorimotor control. ${ }^{15-}$ 18 Individuals with history of LAS have demonstrated decreased spinal reflex ${ }^{19}$ and corticospinal excitability. ${ }^{20,21}$ Furthermore, motor cortex alterations are present in both the injured and non-injured limbs following ankle joint injury. ${ }^{22}$ These findings suggest some type of SMN reorganization following injury. However, the relationship between sensorimotor network functional connectivity (SMN-FC) and static postural control in injured and non-injured limbs remains unknown. The SMN is often overlooked when determining the relationship between static postural control and sensorimotor function. ${ }^{23}$ Elucidating this relationship in injured and non-injured limbs may provide important information related to SMN communication following LAS.

Our aim was to determine the relationship between SMN-FC and static postural control measures in both the injured and non-injured limbs of older adults with a history of LAS. To execute this aim, we selected four regions of interest (ROI) that have potential links to static postural control: bilateral thalami and bilateral post-central gyri. We hypothesized that older adults with a history of LAS would demonstrate a relationship between contralateral SMN-FC and static postural stability in the non-injured, but not the injured, limb.

\section{Results}

Anthropometric and postural control data for all participants are listed in Table 1. 
Table 1

Participant anthropometrics and postural control data: mean (SD)

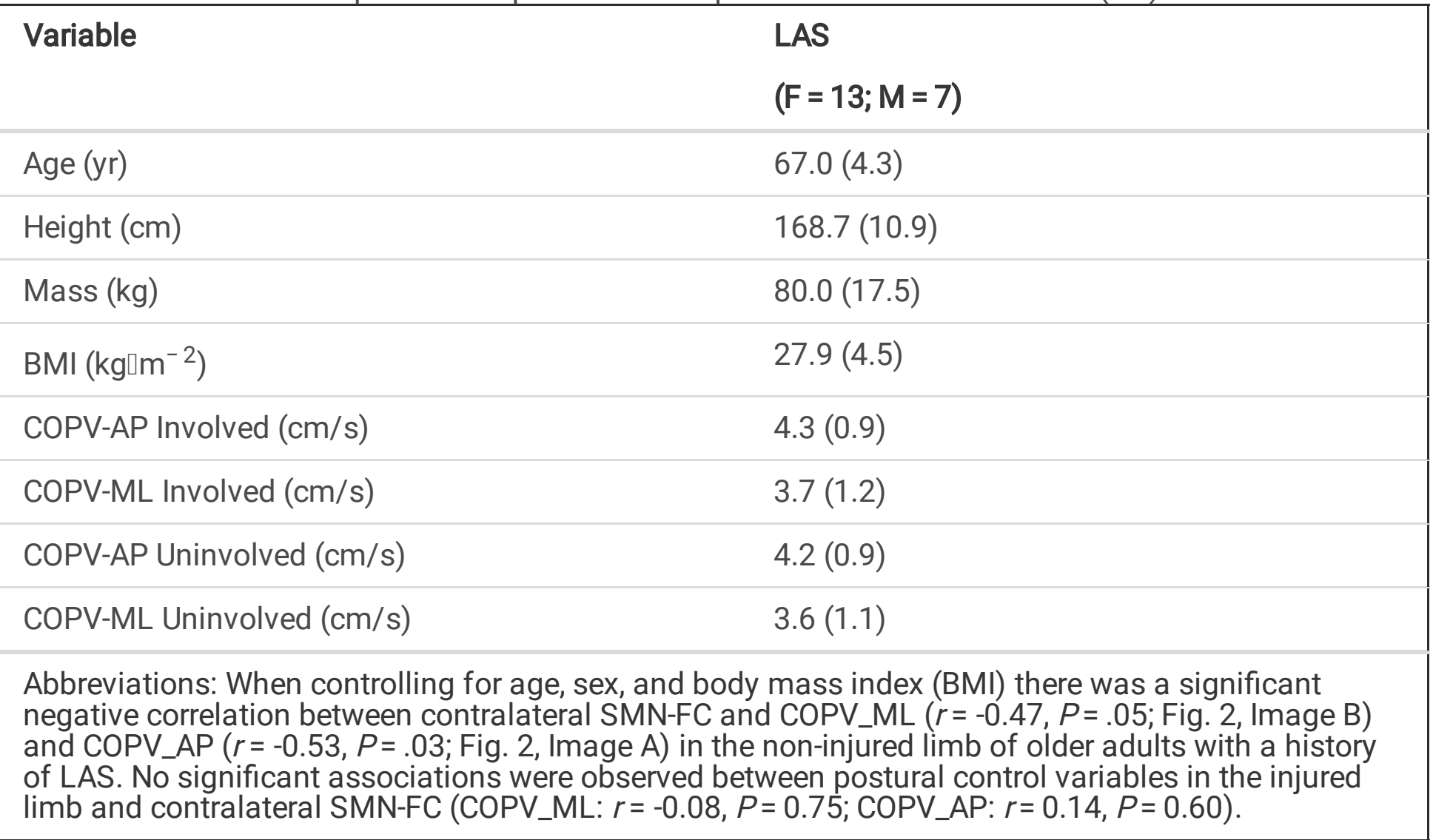

\section{Discussion}

The present study represents a novel investigation into the relationship between CNS connectivity and history of LAS. Specifically, we aimed to determine the relationship between SMN-FC and measures of static postural control in injured and non-injured limbs of individuals with a history of LAS. The results indicated a negative relationship between contralateral SMN-FC and COPV in the non-injured limb of older adults with history of LAS. Further, no relationship was observed between contralateral SMN-FC and COPV in the injured limb of older adults with history of LAS. These findings support our hypothesis and suggest an altered signal fidelity in afferent portions of the SMN related to static postural control in the injured limb. The details and implications of these findings are discussed below.

We observed a potential dissociation between SMN-FC and static postural control in the injured, but not the non-injured, limb of older adults with a history of LAS. Altered CNS sensorimotor communication has been observed in both the injured and non-injured limbs following musculoskeletal injury. ${ }^{20,24,25}$ Both findings suggest a reorganization of cortical communication following peripheral injury. Our findings demonstrate an unspecified loss of signal fidelity, or aberrant neurologic communication, at some point along the afferent pathway. This potential loss of signal fidelity may help to further explain why individuals with functionally unstable ankles are unable to discriminate afferent input related to load when compared to healthy controls. ${ }^{26}$ Taken together, peripheral musculoskeletal injury may lead to maladaptive neuroplastic changes in the CNS. ${ }^{27,28}$ 
Neuroplastic changes are common following peripheral injury. ${ }^{27}$ Greater variability in supplementary motor cortex activation has been observed during a single limb balance task in individuals with a history of LAS. ${ }^{29}$ What remains a mystery is whether this finding is a downstream effect of altered afferent signal fidelity. Interestingly, individuals with history of LAS exhibit peripheral alterations in plantar cutaneous somatosensation ${ }^{15}$, lateral ligament deafferentation ${ }^{30}$, and diminished kinesthesia and joint position sense. ${ }^{31-33}$ Further, articular deafferentation following a LAS may alter proprioceptive feedback to the CNS, creating a loss of signal fidelty. ${ }^{30,34}$ Our findings provide additional support for a potential change in the communication of afferent signal, as the level of functional connectivity is proposed to reflect the amount of signaling exchange between network nodes. ${ }^{35}$

While our findings demonstrate altered SMN communication, the underlying mechanisms remain unknown. Altered SMN connectivity could be the cause or the result of musculoskeletal injury. For example, athletes who go on to experience an ACL injury exhibited differences in pre-injury FC compared to those who did not experience an ACL injury during their sport season. Such findings suggest that preexisting levels of CNS communication may predispose individuals to musculoskeletal injury. Our findings provide additional insight into the existence of different levels of connectivity within SMN nodes that govern injured and non-injured limbs. Taken together, it appears that altered SMN-FC is related to peripheral musculoskeletal injury at multiple lower extremity joints.

Importantly, any predisposition to musculoskeletal injury may be enhanced by age-related declines in postural control and/or FC. Older adults show declines in postural control in conjunction with naturally occurring age-related alterations in FC. ${ }^{36,37}$ For example, older adults typically demonstrate a larger degree of excursion and greater variability of COP, resulting in less stable balance. ${ }^{38}$ Neurophysiological evidence has found increased sway in the ML direction to be indicative of fall risk and age-related disease in older adults. ${ }^{39,40}$ Additionally, age-related decreases in SMN-FC are present in otherwise healthy older adults. ${ }^{41}$ Taken together, the additive effect of age-related declines in postural control and FC may place older adults at an increased risk of falls. Although we did not examine fall risk specifically, reduced afferent processing is ubiquitous in this population ${ }^{42}$ and has been correlated with an increased risk of falling. ${ }^{43}$

The present study has several caveats that warrant further investigation. First, the cross-sectional nature of our study limits the ability to draw causal conclusions. Future studies should incorporate longitudinal designs to determine the degree to which SMN-FC interacts with LAS across the life span. Second, it is difficult to generalize findings since time of initial ankle sprain, severity of ankle sprain, and previous history of rehabilitation following initial LAS are unknown. Third, we utilized a self-reported questionnaire and interview to identify individuals with history of LAS, which may have provided limited information to the overall impact of their LAS. Future studies should include multiple questionnaires and clinical tests to minimize recall bias. Future studies should also explore measures of structural and functional connectivity, as well as potential associations between postural control and white matter microstructure within sensory pathways. White matter microstructure, or anatomical connectivity, contributes to FC and 
is related to static postural control. ${ }^{44,45}$ Finally, we recognize our relatively healthy sample prevents us from generalizing findings to all older adults with history of LAS.

In conclusion, our findings demonstrate a significant relationship between contralateral SMN-FC and COPV in both the AP and ML direction in the non-injured, but not the injured, limb of older adults with history of LAS. This provides preliminary evidence for future studies to determine if LAS leads to alterations in SMN-FC that may have a negative impact on postural control. Additionally, future research should aim to unearth the mechanisms explaining these relationships and to track longitudinal changes in postural control and SMN-FC to enhance our understanding of mechanisms that contribute to age- and injury-related declines in postural control.

\section{Methods}

\section{Study Design}

Using a cross-sectional design, correlations were examined between FC and balance outcomes in older adults with previous history of LAS. The experimental protocol outlined in this manuscript was approved by the University of Kentucky institutional review board. The methods of this study were carried out in accordance with the Declaration of Helsinki. Participants reported to the research laboratory for a single testing session. All participants read and signed an informed consent approved by the University's institutional review board.

\section{Participants}

Twenty older adults with a self-reported previous history of LAS were recruited from local and university communities (Table 1). These individuals had a history of sustaining at least one significant LAS before the age of 35 that resulted in swelling, pain, and a temporary loss of function. No participant had acutely sprained his or her ankle in the three months prior to testing. The previously injured limb was considered the 'testing' limb. In the event a participant reported having a bilateral ankle sprain history, the dominant limb was used as the testing limb. Other than history of LAS, all participants were in good health and had no history of 1) diagnosed balance or vestibular disorders; 2) self-reported low back pain in the past 12 months or history of scoliosis or spondylitis; 3 ) self-reported concussion in the past 12 months; 4) diagnosed cardiopulmonary disorder; 5 ) history of any self-reported musculoskeletal injury or fracture in either the upper or lower extremity; 6 ) any diagnosed neurovascular disorders. Additional exclusion criteria for MRI scanning procedures included: 1 ) history of major neurological disorder (i.e., stroke, seizures) and 2) presence of metal fragments and/or metallic implants that could cause bodily harm or risk disrupting the magnetic field. ${ }^{46}$

\section{Procedures}

\section{MRI Acquisition}


MRI data were acquired utilizing the methodology outlined by Johnson et al. ${ }^{47}$ Data were collected on a 3 Tesla Siemens PRISMA scanner at the University of Kentucky. A 64-channel head coil was used to collect all neuroimaging data. Two primary imaging sequences were collected for each participant in this study: 1) a high-resolution, $T 1$-weighted sequence for subsequent localization of resting-state activity in standard stereotactic space, and 2) T2*-weighted images sensitive to resting fluctuations in bloodoxygen-level-dependent (BOLD) signal.

The high-resolution 3D anatomic images were acquired using a magnetization-prepared rapid gradientecho (MPRAGE) sequence with the following parameters: echo time (TE) $2.26 \mathrm{~ms}$, repetition time (TR) $2530 \mathrm{~ms}$, field of view (FOV) of $256 \mathrm{~mm}$, flip angle (FA) of $7^{\circ}$, and voxel size of $1 \times 1 \times 1 \mathrm{~mm}$. T2*-weighted images sensitive to changes in BOLD were acquired with the following parameters: TE $30 \mathrm{~ms}$, TR 2000 $\mathrm{ms}, \mathrm{FOV}=224 \mathrm{~mm},(\mathrm{FA})$ of $76^{\circ}$, and voxel size of $3.5^{3} \mathrm{~mm}^{3}$.

\section{Resting-State Processing}

Intrinsic connectivity during resting-state fMRI was assessed via independent component analysis (ICA) using FMRIB's Software Library (FSL) Multivariate Exploratory Linear Optimized Decomposition into Independent Components (MELODIC) tool (http://fsl.fmrib.ox.ac.uk/fsl/fsl-4.1.9/melodic/index.html). ${ }^{47}$ ICA is a statistical technique that decomposes a summative signal into independent additive spatiotemporal subcomponents. MELODIC's multi-session temporal concatenation option was employed to allow for the identification of group-level spontaneous intrinsic brain networks, or subcomponents.

The resting-state data were brain-extracted ${ }^{48}$, motion corrected to the median functional image using bspline interpolation, temporally filtered with a 100 second high-pass filter, and spatially smoothed with a 7 $\mathrm{mm}$ full width at half maximum (FWHM) kernel. The previously skull stripped T1-weighted anatomic volumes (FSL's brain extraction tool, BET) were registered to the standard space T1 MNI $2 \times 2 \times 2 \mathrm{~mm}$ template with FSL's Non-linear Image Registration Tool (FNIRT;

http://www.fmrib.ox.ac.uk/analysis/techrep). Each participant's median functional image was then coregistered to their anatomical volume and warped to standard space using the non-linear warping matrix generated during the transformation of the anatomical volume to standard space. All resulting functional images were interpolated to $2 \times 2 \times 2 \mathrm{~mm}$ resolution for group-level network identification.

The SMN component was identified at the group level (component 1) and thresholded at a significance level of $Z>3.1$. The thresholding yielded a SMN map composed of bilateral pre- and post-central gyri and bilateral thalami (Fig. 1).

The thresholded map was then multiplied by left and right hemisphere masks to isolate ipsilateral and contralateral connectivity maps for injured and uninjured limbs. FSL's clustertool was then used to identify peaks in each hemisphere. The Harvard-Oxford Cortical Structure Atlas was then used to identify peaks in both the left and right postcentral gyri. Afferent structures were of primary interest due to peripheral deafferentation following injury. Peak coordinates for bilateral postcentral gyri and thalami can be found in Table 2 . 
Table 2

SMN Peak Coordinates

\begin{tabular}{|lccc|}
\hline Region & \multicolumn{3}{l|}{ MNI Coordinates in Voxels } \\
\hline & $x$ & $y$ & $z$ \\
\hline Left postcentral gyrus & 65 & 54 & 59 \\
\hline Left thalamus & 50 & 53 & 35 \\
\hline Right postcentral gyrus & 26 & 49 & 64 \\
\hline Right thalamus & 38 & 53 & 37 \\
\hline $\begin{array}{l}\text { Peak coordinates in the SMN thresholded at Z = 3.1 } \\
\text { SMN, sensorimotor network }\end{array}$ \\
\hline
\end{tabular}

Peak coordinates were then transformed to native space using FSL's img2imgcoord tool. Next, fslmaths was used to create $4 \mathrm{~mm}$ spheres and a single network mask in native space. Finally, Analysis of Functional Neuroimages' 3dNetCorrtool was used to pull Pearson r and Fisher-transformed Z-scores for individual network correlations. Higher correlation coefficients are indicative of more synchronous communication between ROls.

\section{Balance Assessment}

Following resting-state fMRI data collection, single limb static postural control was assessed on a force platform utilizing the methodology outlined by Terada et al. ${ }^{44}$ Participants performed three eyes-open trials of a 20-second single-limb balance task on a level surface on both the injured and non-injured limbs. Order of limb testing was randomized. Center of pressure velocity (COPV) measures were assessed using a portable force platform (Accusway Plus, AMTI, Watertown, MA) integrated with Balance Clinic software (AMTI) at a sampling rate of $100 \mathrm{~Hz}$. A custom-made MATLAB code was used to calculate center of pressure (COP) trajectories in the anteroposterior (COPV_AP) and mediolateral (COPV_ML) directions. The COPV time series data were filtered with a low pass, fourth-order Butterworth filter set at a cutoff frequency of $5 \mathrm{~Hz} .{ }^{10}$ Lower values of COPV are indicative of better static postural control.

Participants were asked to stand barefoot on the testing limb in single-limb stance in the middle of the force plate, with their hands on their hips and foot flat. Three practice trials preceded test trials. During the testing trials, participants were instructed to maintain their single-limb posture with eyes open for 20seconds while COP data were collected. A trial was discarded if: 1) the non-testing limb made contact on the force platform or the stance limb; 2) participants hopped or took a step with the stance limb; 3) participant lifted their forefoot or heel from the force plate; and/or 4) they removed their hands from their hips. $^{44}$

\section{Statistical Analysis}


Both sex and BMI have been shown to influence static postural control, so age, sex and BMI were entered

as nuisance covariates $(P<0.05) .{ }^{49,50}$ Partial correlations with standardized residuals were performed to assess the relationship of contralateral SMN-FC and the injured and uninjured side COPV for both the AP and $\mathrm{ML}$ directions. Pearson product moment correlation coefficients $(r)$ were interpreted as weak (0.000.40), moderate (0.41-0.69), or strong (0.70-1.00). ${ }^{51}$ An a priori alpha level was set at $P<0.05$ using the Statistical Package for the Social Sciences (version 24.0; SPSS Inc., Chicago, IL.) for all statistical tests.

\section{Declarations}

\section{COMPETING INTERESTS}

The authors declare no competing interests.

\section{AUTHOR CONTRIBUTION STATEMENT}

KB and NJ wrote the main manuscript and created the figures and tables. MT, PG, and KK recruited, screened, and enrolled all participants. All authors reviewed the manuscript and provided in-depth feedback throughout its' creation.

\section{References}

1. Cameron, K. L., Owens, B. D. \& DeBerardino, T. M. Incidence of ankle sprains among active-duty members of the United States Armed Services from 1998 through 2006. Journal of athletic training. 45, 29-38 (2010).

2. Gribble, P. A. et al. Selection criteria for patients with chronic ankle instability in controlled research: a position statement of the International Ankle Consortium. J Orthop Sports Phys Ther. 43, 585-591 https://doi.org/10.2519/jospt.2013.0303 (2013).

3. Anandacoomarasamy, A. \& Barnsley, L. Long term outcomes of inversion ankle injuries. British journal of sports medicine. 39, e14-e14 (2005).

4. Gribble, P. A. et al. 2016 consensus statement of the International Ankle Consortium: prevalence, impact and long-term consequences of lateral ankle sprains. British journal of sports medicine. 50, 1493-1495 (2016).

5. Hertel, J. \& Corbett, R. O. An updated model of chronic ankle instability. Journal of athletic training. 54, 572-588 (2019).

6. Needle, A. R., Lepley, A. S. \& Grooms, D. R. Central nervous system adaptation after ligamentous injury: a summary of theories, evidence, and clinical interpretation. Sports Med. 47, 1271-1288 (2017).

7. Wikstrom, E. A., Naik, S., Lodha, N. \& Cauraugh, J. H. Bilateral balance impairments after lateral ankle trauma: a systematic review and meta-analysis. Gait \& posture. 31, 407-414 (2010). 
8. Terada, M. et al. Quantifying levels of function between different subgroups of chronic ankle instability. Scandinavian journal of medicine \& science in sports. 27, 650-660 (2017).

9. Evans, T., Hertel, J. \& Sebastianelli, W. Bilateral deficits in postural control following lateral ankle sprain. Foot \& ankle international. 25, 833-839 (2004).

10. McKeon, P. O. \& Hertel, J. Spatiotemporal postural control deficits are present in those with chronic ankle instability. BMC musculoskeletal disorders. 9, 76 (2008).

11. Terada, M., Kosik, K., Johnson, N. \& Gribble, P. Altered postural control variability in older-aged individuals with a history of lateral ankle sprain. Gait \& posture. 60, 88-92 (2018).

12. Khanna, N., Altmeyer, W., Zhuo, J. \& Steven, A. Functional neuroimaging: fundamental principles and clinical applications. The neuroradiology journal. 28, 87-96 (2015).

13. Goble, D. J. et al. Brain activity during ankle proprioceptive stimulation predicts balance performance in young and older adults. Journal of Neuroscience. 31, 16344-16352 (2011).

14. Diekfuss, J. A. et al. Does brain functional connectivity contribute to musculoskeletal injury? A preliminary prospective analysis of a neural biomarker of ACL injury risk. Journal of science and medicine in sport. 22, 169-174 (2019).

15. Hoch, M. C., McKeon, P. O. \& Andreatta, R. D. Plantar vibrotactile detection deficits in adults with chronic ankle instability.Medicine and science in sports and exercise44 (2012).

16. McKeon, J. M. M. \& McKeon, P. O. Evaluation of joint position recognition measurement variables associated with chronic ankle instability: a meta-analysis. Journal of athletic training. 47, 444-456 (2012).

17. Hoch, M. C. \& McKeon, P. O. Peroneal reaction time after ankle sprain: a systematic review and metaanalysis. Medicine and Science in Sports and Exercise46 (2014).

18. Wikstrom, E. A., Naik, S., Lodha, N. \& Cauraugh, J. H. Balance capabilities after lateral ankle trauma and intervention: a meta-analysis. Medicine \& Science in Sports \& Exercise. 41, 1287-1295 (2009).

19. Kim, K. M. et al. Changes in Spinal and Corticospinal Excitability in Patients with Chronic Ankle Instability: A Systematic Review with Meta-Analysis. Journal of clinical medicine. 8, 1037 (2019).

20. McLeod, M. M., Gribble, P. A. \& Pietrosimone, B. G. Chronic ankle instability and neural excitability of the lower extremity. Journal of athletic training. 50, 847-853 (2015).

21. Needle, A. R. et al. The relationship between the sensory responses to ankle-joint loading and corticomotor excitability. International Journal of Neuroscience. 128, 435-441 (2018).

22. Kosik, K. B., Terada, M., Drinkard, C. P., McCann, R. S. \& Gribble, P. A. Potential Corticomotor Plasticity in Those with and without Chronic Ankle Instability. Medicine and science in sports and exercise. 49, 141-149 (2017).

23. Ivanenko, Y. \& Gurfinkel, V. S. Human postural control. Frontiers in neuroscience. 12, 171 (2018).

24. Pietrosimone, B. G. \& Gribble, P. A. Chronic ankle instability and corticomotor excitability of the fibularis longus muscle. Journal of athletic training. 47, 621-626 (2012). 
25. Héroux, M. E. \& Tremblay, F. Corticomotor excitability associated with unilateral knee dysfunction secondary to anterior cruciate ligament injury. Knee Surg. Sports Traumatol. Arthrosc. 14, 823-833 (2006).

26. Needle, A. R. et al. Decoupling of laxity and cortical activation in functionally unstable ankles during joint loading. European journal of applied physiology. 114, 2129-2138 (2014).

27. Navarro, X., Vivó, M. \& Valero-Cabré, A. Neural plasticity after peripheral nerve injury and regeneration. Progress in neurobiology. 82, 163-201 (2007).

28. Pelletier, R., Higgins, J. \& Bourbonnais, D. Is neuroplasticity in the central nervous system the missing link to our understanding of chronic musculoskeletal disorders? BMC musculoskeletal disorders. 16, 1-13 (2015).

29. Rosen, A. B. et al. Alterations in Cortical Activation Among Individuals With Chronic Ankle Instability During Single-Limb Postural Control. Journal of athletic training. 54, 718-726 (2019).

30. Freeman, M. Instability of the foot affer injuries to the lateral ligament of the ankle. The Journal of bone and joint surgery. British volume. 47, 669-677 (1965).

31. Lentell, G. et al. The contributions of proprioceptive deficits, muscle function, and anatomic laxity to functional instability of the ankle. Journal of Orthopaedic \& Sports Physical Therapy. 21, 206-215 (1995).

32. Forkin, D. M., Koczur, C., Battle, R. \& Newton, R. A. Evaluation of kinesthetic deficits indicative of balance control in gymnasts with unilateral chronic ankle sprains. Journal of Orthopaedic \& Sports Physical Therapy. 23, 245-250 (1996).

33. Gross, M. T. Effects of recurrent lateral ankle sprains on active and passive judgments of joint position. Physical therapy. 67, 1505-1509 (1987).

34. Gutierrez, G. M., Kaminski, T. W. \& Douex, A. T. Neuromuscular control and ankle instability. Pm\&r. 1, 359-365 (2009).

35. Van Den Heuvel, M. P. \& Pol, H. E. H. Exploring the brain network: a review on resting-state fMRI functional connectivity. European neuropsychopharmacology. 20, 519-534 (2010).

36. Farras-Permanyer, L. et al. Age-related changes in resting-state functional connectivity in older adults. Neural regeneration research. 14, 1544 (2019).

37. Varangis, E., Habeck, C., Razlighi, Q. \& Stern, Y. The effect of aging on resting state connectivity of predefined networks in the brain. Frontiers in aging neuroscience. 11, 234 (2019).

38. Van Emmerick, R., McDermott, B., Haddad, J., Van Wegen, E. \& Baird, J.128-129(HUMAN KINETICS PUBL INC 1607 N MARKET ST, CHAMPAIGN, IL 61820 - 2200 USA).

39. Maki, B. E., Holliday, P. J. \& Topper, A. K. A prospective study of postural balance and risk of falling in an ambulatory and independent elderly population. Journal of gerontology. 49, M72-M84 (1994).

40. Mitchell, S. L., Collin, J. J., De Luca, C. J., Burrows, A. \& Lipsitz, L. A. Open-loop and closed-loop postural control mechanisms in Parkinson's disease: increased mediolateral activity during quiet standing. Neuroscience letters. 197, 133-136 (1995). 
41. Huang, C. C. et al. Age-related changes in resting-state networks of a large sample size of healthy elderly. CNS Neuroscience \& Therapeutics. 21, 817-825 (2015).

42. Maki, B. E. et al. Preventing falls in older adults: new interventions to promote more effective changein-support balance reactions. Journal of electromyography and kinesiology. 18, 243-254 (2008).

43. Lord, S. R., Ward, J. A., Williams, P. \& Anstey, K. J. Physiological factors associated with falls in older community-dwelling women. Journal of the American Geriatrics Society. 42, 1110-1117 (1994).

44. Terada, M., Johnson, N., Kosik, K. \& Gribble, P. Quantifying Brain White Matter Microstructure of People with Lateral Ankle Sprain. Medicine and science in sports and exercise. 51, 640-646 (2019).

45. Peterson, D. S., Gera, G., Horak, F. B. \& Fling, B. W. Corpus callosum structural integrity is associated with postural control improvement in persons with multiple sclerosis who have minimal disability. Neurorehabilitation and neural repair. 31, 343-353 (2017).

46. Johnson, N. F., Kim, C. \& Gold, B. T. Socioeconomic status is positively correlated with frontal white matter integrity in aging. Age. 35, 2045-2056 (2013).

47. Johnson, N. F. et al. Cardiorespiratory fitness modifies the relationship between myocardial function and cerebral blood flow in older adults. Neuroimage. 131, 126-132 (2016).

48. Smith, S. M. Fast robust automated brain extraction. Human brain mapping. 17, 143-155 (2002).

49. Carral, J. M. C., Ayán, C., Sturzinger, L. \& Gonzalez, G. Relationships between body mass index and static and dynamic balance in active and inactive older adults. Journal of geriatric physical therapy. 42, E85-E90 (2019).

50. Torres, S. F., Reis, J. G. \& Abreu, D. C. C. d. Influence of gender and physical exercise on balance of healthy young adults. Fisioterapia em Movimento. 27, 399-406 (2014).

51. Lomax, R. G. Statistical concepts: A second course (Lawrence Erlbaum Associates Publishers, 2007).

\section{Figures}




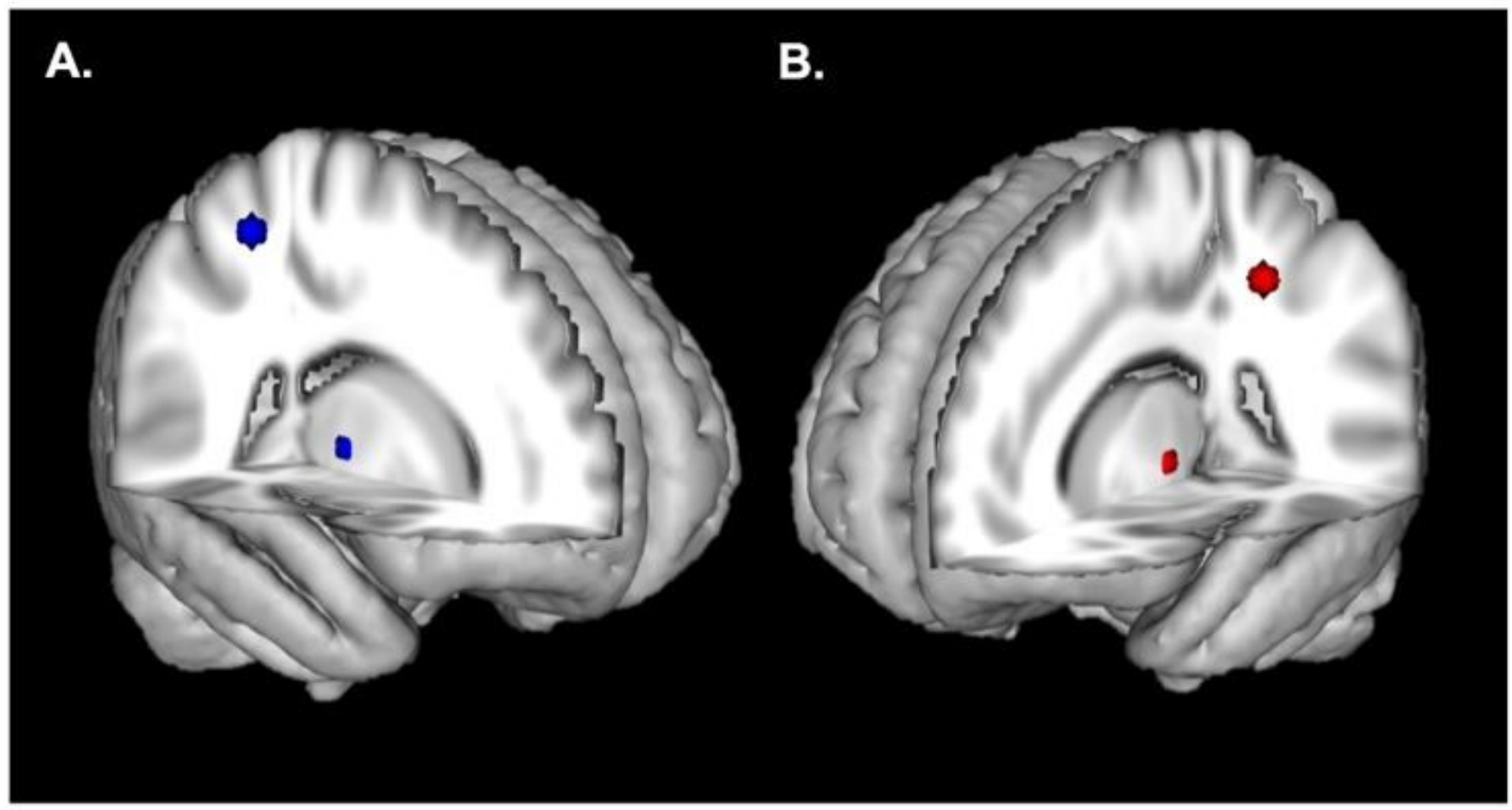

\section{Figure 1}

Sensorimotor Network Spherical Peaks. Right (A; blue) and left (B; red) postcentral gyri and thalamic spherical peaks. The anatomic underlay used for illustration is the MNI152 T1-weighted $2 \mathrm{~mm}$ brain.
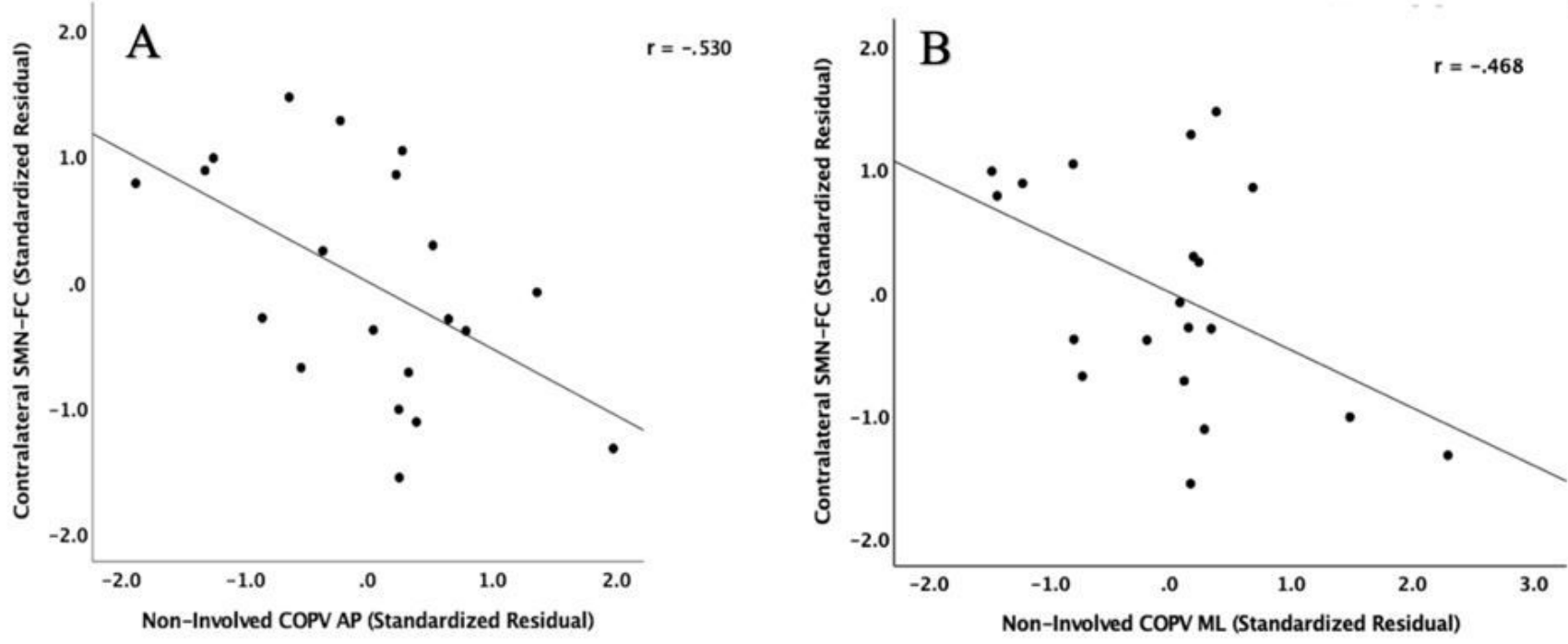

\section{Figure 2}

Image A: Controlling for age, sex, and BMI there was a moderate negative correlation between contralateral SMN-FC and COPV_AP $(r=-0.53, P=.03)$. Image $B$ : Controlling for age, sex, and BMI there was a moderate negative correlation between contralateral SMN-FC and COPV_ML $(r=-0.47, P=.05)$. 